\title{
CRAMÉR RAO BOUND ANALYSIS OF JOINT B1/T1 MAPPING METHODS IN MRI
}

\author{
Amanda Funai and Jeffrey A. Fessler \\ EECS Dept., The University of Michigan \\ \{afunai,fessler\}@umich.edu
}

\begin{abstract}
In MRI, RF field inhomogeneity $\left(B_{1}\right)$ and relaxation effects ( $\left.T_{1}\right)$ significantly affect both $B_{1}$ and $T_{1}$ mapping. Simultaneous joint estimation of both $B_{1}$ and $T_{1}$ has the potential to greatly improve both $B_{1}$ and $T_{1}$ estimation. This paper analyzes the Cramér Rao Bound for joint $B_{1}, T_{1}$ estimation using common $B_{1}$ and $T_{1}$ pulse sequences. This analysis aids choosing pulse sequences and parameters given desired levels of $B_{1}$ and $T_{1}$ accuracy and the inherent trade off between the two mappings.
\end{abstract}

Index Terms - Magnetic resonance imaging, B1 mapping, T1 mapping, Cramer Rao Bound

\section{INTRODUCTION}

In MRI, maps of the $B_{1}^{+}$field strength, called a $B_{1}^{+}$map, and of the longitudinal relaxation time $T_{1}$, called a $T_{1}$ map, are essential in many situations. $B_{1}^{+}$maps are required for parallel transmit excitation RF pulse design (using a coil array) [1] and for pre-scan calibration at high fields $(\geq 3 \mathrm{~T})$ where large $B_{1}^{+}$inhomogeneity creates spatially varying signal and contrast. [2]. Fast, accurate, and precise mapping of $T_{1}$ has many applications: finding tumors or assessing organs and function, perfusion imaging, diagnosing disease, quantifying myocardial blood flow, and preparing navigation and visualization tools for surgery.

$T_{1}$ and $B_{1}^{+}$mapping are closely linked. Relaxation effects, unless properly accounted for, can confound $B_{1}^{+}$estimation. Using a very large repetition time (e.g., TR $>5 T_{1}$ in the popular double angle method) removes any $T_{1}$ dependence from the acquired images, but scans are then undesirably slow for in vivo imaging. While fast and improved methods for the double angle method use scan time more efficiently [2], recent model-based $B_{1}^{+}$mapping estimation methods [3] incorporate $T_{1}$ estimation to account for these relaxation effects.

$T_{1}$ mapping can also be adversely effected by $B_{1}^{+}$inhomogeneity, especially using gradient echo and spin echo acquisitions with a short TR. Steady-state incoherent (SSI) imaging, a very popular fast imaging method that can be used

Supported in part by an NSF GRF and by NIH grants CA 87634 and NS 058576 . in $T_{1}$ mapping, is especially sensitive [4]. $B_{1}^{+}$inhomogeneity causes large inaccuracies in uncorrected $T_{1}$ mapping [5].

Recently, methods have been developed that jointly estimate both $B_{1}^{+}$and $T_{1}$ (such as [3]). Making an informed choice between the wide variety of pulse sequences where relaxation effects and $B_{1}^{+}$inhomogeneity feature prominently remains an open problem. Analysis of the accuracy and precision possible in $B_{1}^{+}$and $T_{1}$ estimates and the inherent trade offs can aid this selection.

In this paper, we first construct a general model for joint $B_{1}^{+}, T_{1}$ mapping. We then use the Cramér Rao Bound to analyze the lowest possible variance for unbiased joint estimation of $B_{1}^{+}$and $T_{1}$ using several specific pulse sequences. We investigate the variance of both estimates over a range of $B_{1}^{+}$ and $T_{1}$ values. We also use this analysis to help optimize timing and flip angle parameters for each pulse sequence. This analysis extends the large body of research on optimization of parameters and precision for $T_{1}$ estimation (e.g., [6]) to include joint $B_{1}^{+}$and $T_{1}$ estimation. The trade offs and analysis from this paper allows comparison of pulse sequences depending on the particular required accuracy for both $B_{1}^{+}$ and $T_{1}$.

\section{JOINT ESTIMATION MODEL}

\subsection{General Joint Estimation Model}

Most $B_{1}^{+}$or $T_{1}$ mapping pulse sequences can be formulated using the following general model. Let the measured value of a single voxel for the $i$ th scan be given by:

$$
y_{i}=m_{0} F\left(\frac{T_{R i}}{T_{1}}, \alpha_{i} b\right)+\epsilon_{i},
$$

where $m_{0}$ is the nominal voxel magnetization dependent on $T_{E}$ (the echo time) and $T_{2} . T_{1}$ is the longitudinal relaxation constant, $b$ is the RF field strength at this voxel, $T_{R i}$ is the repetition time for a specific pulse sequence, and $\alpha_{i}$ is the relative amplitude of the RF pulse, where the product $\alpha_{i} b$ specifies the flip angle in a given voxel. The unitless function $F$ describes the MRI scan signal value variation independent of $T_{E}$ and $T_{2}$ based on the individual pulse sequence and scan parameters and is defined in Section $\S 2.2$ for three specific models. The full data $Y=\left(y_{1}, \cdots, y_{N}\right)$ consists of $N$ scans where 
either $T_{R i}$ or $\alpha_{i}$ is varied. For example, for the double angle $B_{1}^{+}$mapping method, $N=2$ and $\alpha_{2}=2 \alpha_{1}$ and $T_{R 1}=T_{R 2}$. $\epsilon_{i}$ is modeled as white, Gaussian noise. While magnitude images suffer from Rician noise, we model complex scans with true Gaussian noise. We assume that $\sigma_{\epsilon_{i}}=\sigma_{0} \forall i$.

The Cramér Rao Bound (CRB) expresses the lowest achievable variance possible for an unbiased estimator for a given model. Although practical estimators are often biased (e.g., through smoothing or filtering the data or using approximations to the model), the bound quantifies the estimator variance and captures the coupling effects between the two unknown parameters. Because $\boldsymbol{\theta}=\left(b, T_{1}\right)$, the multiple parameter CRB must be used. In that case, the matrix CRB is

$$
\operatorname{Cov}_{\boldsymbol{\theta}}\{\hat{\boldsymbol{\theta}}\} \geq \boldsymbol{J}^{-1}(\boldsymbol{\theta})
$$

where the Fisher information matrix is

$$
\boldsymbol{J}(\boldsymbol{\theta})=\mathrm{E}\left[\left[\nabla_{\boldsymbol{\theta}} \ln \mathrm{p}(Y ; \boldsymbol{\theta})\right]\left[\nabla_{\boldsymbol{\theta}} \ln \mathrm{p}(Y ; \boldsymbol{\theta})\right]^{T}\right] .
$$

The Fisher information $\boldsymbol{J}$ is a $2 \times 2$ matrix with entries:

$$
\begin{aligned}
J_{11} & =\frac{1}{\sigma^{2}} \sum_{i}\left(\frac{\partial}{\partial b} \bar{y}_{i}\right)^{2} \\
J_{12} & =J_{21}=\frac{1}{\sigma^{2}} \sum_{i}\left(\frac{\partial}{\partial b} \bar{y}_{i}\right)\left(\frac{\partial}{\partial T_{1}} \bar{y}_{i}\right) \\
J_{22} & =\frac{1}{\sigma^{2}} \sum_{i}\left(\frac{\partial}{\partial T_{1}} \bar{y}_{i}\right)^{2},
\end{aligned}
$$

where $\bar{y}_{i}$ is the expected value of $y_{i}$. We define $\phi_{i} \triangleq \alpha_{i} b$ (tip angle) and $\gamma_{i} \triangleq \frac{T_{R i}}{T_{1}}$. The derivatives of the general model (1) then are:

$$
\begin{aligned}
\frac{\partial}{\partial T_{1}} \bar{y}_{i} & =-M_{0} \cdot \frac{T_{R i}}{T_{1}^{2}} \cdot F^{01}\left(\phi_{i}, \gamma_{i}\right) \\
\frac{\partial}{\partial b} \bar{y}_{i} & =M_{0} \cdot \alpha_{i} \cdot F^{10}\left(\phi_{i}, \gamma_{i}\right),
\end{aligned}
$$

where $F^{10}$ and $F^{01}$ denote partial derivatives with respect to the first and second arguments of $F$ respectively. Then,

$$
\begin{aligned}
\sigma_{b} \triangleq \sqrt{C R B(b)} & =\sqrt{\left[\boldsymbol{J}^{-1}(\boldsymbol{\theta})\right]_{11}} \\
\sigma_{T_{1}} \triangleq \sqrt{C R B\left(T_{1}\right)} & =\sqrt{\left[\boldsymbol{J}^{-1}(\boldsymbol{\theta})\right]_{22}} .
\end{aligned}
$$

In this paper, we calculate the CRB for several specific models over a wide range of input parameters and optimize the scan parameters.

\subsection{Specific Joint Estimation Models}

For joint estimation model selection, we consider three main pulse sequences, with their corresponding models for $F$ in (1). First, the SSI model [4] where

$$
F_{i}^{\mathrm{SSI}}=\frac{\left(1-e^{-\gamma_{i}}\right) \sin \left(\phi_{i}\right)}{1-e^{-\gamma_{i}} \cos \left(\phi_{i}\right)} .
$$

This pulse sequence is used commonly for $T_{1}$ mapping by varying $\alpha_{i}$ although $T_{R i}$ can also be varied; this sequence also has been used successfully for solo $B_{1}^{+}$mapping as in [7].

Second, we consider the Brunner-Pruessmann method (BP) used in [3] using a non-selective, spoiled prepulse with a varying flip angle $\left(\phi_{i}\right)$ followed by a slice excitation with a flip angle $\beta b$. As in [3], we set $\Delta=.05 \mathrm{~ms}$ and $\beta b=20^{\circ}$ to reduce the number of parameters to optimize. We define: $\eta \triangleq \frac{\Delta}{T_{1}}$. We also ignore any $B_{0}$ inhomogeneity and use the following model:

$$
\begin{aligned}
F_{i}^{\mathrm{BP}}= & \cos \left(\phi_{i}\right) \sin (\beta b) \cdot \\
& \frac{\cos \left(\phi_{i}\right) e^{-\eta}\left(1-e^{\left.-\gamma_{i}-\eta\right)}\right)+1-e^{-\eta}}{1-\cos \left(\phi_{i}\right) \cos (\beta b) e^{-\gamma_{i}}} .
\end{aligned}
$$

Third, we consider pulse sequence used in the Actual Flip Angle (AFI) method [8]. When this pulse sequence is used in $B_{1}^{+}$mapping, usually approximations and ratios are used to remove $T_{1}$ dependence in the final $B_{1}^{+}$estimator. However, the signal depends on both $B_{1}^{+}$and $T_{1}$ and is a candidate for joint estimation. This model differs from the previous two in that two repetition times, $T_{R 1}$ and $T_{R 2}$, are used simultaneously in steady state and thus appear in both equations $F_{1}^{A F I}$ and $F_{2}^{A F I}$ as shown below:

$$
\begin{aligned}
& F_{2 i-1}^{A F I}=\sin \left(\phi_{i}\right) \frac{1-e^{-\gamma_{2}}+\left(1-e^{-\gamma_{1}}\right) e^{-\gamma_{2}} \cos \left(\phi_{i}\right)}{1-e^{-\gamma_{1}-\gamma_{2}} \cos ^{2}\left(\phi_{i}\right)} \\
& F_{2 i}^{A F I}=\sin \left(\phi_{i}\right) \frac{1-e^{-\gamma_{1}}+\left(1-e^{-\gamma_{2}}\right) e^{-\gamma_{1}} \cos \left(\phi_{i}\right)}{1-e^{-\gamma_{1}-\gamma_{2}} \cos ^{2}\left(\phi_{i}\right)}(9)
\end{aligned}
$$

\section{CRAMÉR RAO BOUND ANALYSIS AND DISCUSSION}

\subsection{Method and Results}

To compare the models using the CRB, we derived the CRB using implicit differentiation in MATLAB. To enable fair comparison of models using different imaging time, consider that a scan repeated $N$ times gives a standard deviation $\sigma_{0} / \sqrt{N}$. Therefore, we report $\tilde{\sigma}_{b} \triangleq \sigma_{b} \sqrt{\sum_{i} T_{R i}} \frac{m_{0}}{\sigma}$ (compare [6]), defined as the TR Compensated Deviation (TRCD). To make optimization feasible over a very large parameter space, we constrain the search space by requiring that $\alpha_{i}=i \Delta_{\alpha}$ for the SSI (7) and BP (8) models. For the AFI model, we keep $T_{R 1}$ and $T_{R 2}$ constant and set $\alpha_{2 i-1}=\alpha_{2 i}=i \Delta_{\alpha}$. Therefore, we optimize over only 4-5 parameters regardless of the number of scans: $\left(\Delta_{\alpha}, T_{R i}, b, T_{1}\right)$.

The ideal model will have a low $\tilde{\sigma}_{b}$ and $\tilde{\sigma}_{T_{1}}$ and also be relatively insensitive to variation in $B_{1}^{+}$and $T_{1}$. There is a trade off between optimizing both TRCD values; therefore, we use a scalar valued function

$f\left(\Delta_{\alpha}, T_{R}, b, T_{1}\right)=\tilde{\sigma}_{b}\left(\Delta_{\alpha}, T_{R}, b, T_{1}\right)+\tilde{\sigma}_{T_{1}}\left(\Delta_{\alpha}, T_{R}, b, T_{1}\right)$ 
in our optimization to consider the effect of both TRCDs. We seek scan parameters (tip angles and repetition times) whose TRCDs have low variation over a wide range of $T_{1}$ and $B_{1}^{+}$ values. We perform a min-max optimization; we minimize over the set of scan parameters the worst-case (i.e., maximum) $f(\cdot)$ over the range of $B_{1}^{+}$and $T_{1}$ values. This is expressed mathematically as optimizing the following equation:

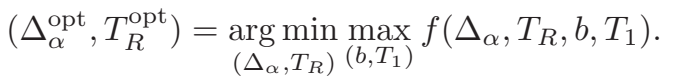

We first find the TRCD over a large parameter space defined by the maximum tip angle $\Delta_{\alpha} \cdot N \in[\pi / 4,9 \pi / 4]$, $T_{R} \in[.1,3], T_{1} \in[.2,1.2]$, and $b \in[.5,2]$; these denote the "search" range. We perform the optimization in (10). The optimal values for our choice of $f$ are shown in Table 1 .

To analyze the trade off between $\tilde{\sigma}_{b}$ and $\tilde{\sigma}_{T_{1}}$, we also find the worst case TRCD values over the range of $B_{1}^{+}$and $T_{1}$. We define

$$
\begin{aligned}
& \tilde{\sigma}_{b}^{\max } \triangleq \max _{b, T_{1}} \tilde{\sigma}_{b}\left(\Delta_{\alpha}, T_{R}, b, T_{1}\right), \\
& \tilde{\sigma}_{T_{1}}^{\max } \triangleq \max _{b, T_{1}} \tilde{\sigma}_{T_{1}}\left(\Delta_{\alpha}, T_{R}, b, T_{1}\right) .
\end{aligned}
$$

We then plot, for each $\tilde{\sigma}_{b}^{\max }$, the lowest achievable $\tilde{\sigma}_{T_{1}}^{\max }$ over $\Delta_{\alpha}$ and $T_{R}$. These plots are shown in Fig. $4(N=4)$ and Fig. $5(N=8)$.

Next, using the optimal parameters $\Delta_{\alpha}^{\mathrm{opt}}$ and $T_{R i}^{\mathrm{opt}}(10)$, we calculate the TRCD over a larger range of $B_{1}^{+}$(keeping the range of $T_{1}$ the same): $b \in[.25, .4]$; this is the "display" range. Now, we can see how robust the optimized parameters are when $B_{1}^{+}$and $T_{1}$ are outside the original search range. We plot, for each $B_{1}^{+}$value in the display range, the maximum $\tilde{\sigma}$ over the $T_{1}$ search range on one set of graphs (e.g., $\tilde{\sigma}_{b}$ in plot $\mathrm{B}$ and $\tilde{\sigma}_{T_{1}}$ in plot D); and also for each $T_{1}$ value in the display range, the maximum $\tilde{\sigma}$ over the $B_{1}^{+}$search range on another set of graphs (e.g., $\tilde{\sigma}_{b}$ in plot $\mathrm{A}$ and $\tilde{\sigma}_{T_{1}}$ in plot $\left.\mathrm{C}\right)$. The graphs are shown in Fig. 1, Fig. 2, and Fig. 3.

Table 1. Optimized scan parameters based on (10)

\begin{tabular}{|c|c|c|c|c|}
\hline Model & $N$ & $\begin{array}{c}\Delta_{\alpha} \text { or } \alpha \\
\text { (radians) }\end{array}$ & $\begin{array}{c}\Delta_{T_{R}} \text { or } T_{R 1} \\
(\mathrm{sec})\end{array}$ & $\begin{array}{c}T_{R 2} \\
(\mathrm{sec})\end{array}$ \\
\hline \hline SSI & 2 & 1.1781 & 0.68 & - \\
SSI & 4 & 1.3744 & 0.68 & - \\
SSI & 8 & 0.8836 & 0.68 & - \\
\hline AFI & 2 & 1.0996 & 0.245 & 0.10 \\
AFI & 4 & 1.3352 & 0.825 & 0.10 \\
AFI & 8 & 1.0603 & 0.68 & 0.10 \\
\hline BP & 2 & 2.2776 & 0.825 & - \\
BP & 4 & 0.9818 & 0.535 & - \\
BP & 8 & 0.8836 & 0.825 & - \\
\hline
\end{tabular}
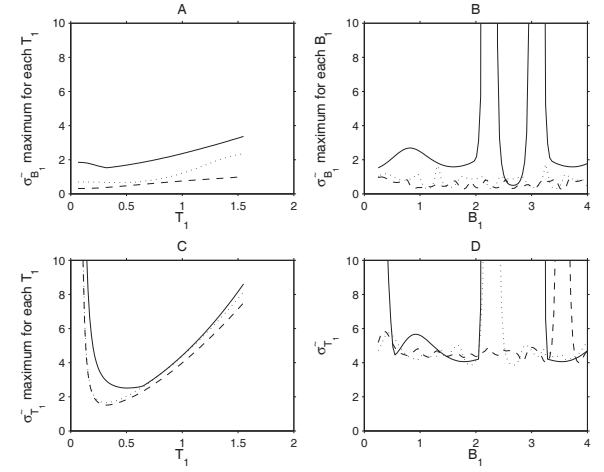

Fig. 1. SSI model, $N=2$ (solid line), 4 (dotted line), 8 (dashed line). We plot, at the optimal parameters in Table 1, the maximum $\tilde{\sigma}_{b}$ for each $T_{1}$ over $B_{1}^{+}$values in the search range (A), the maximum $\tilde{\sigma}_{b}$ for each $B_{1}^{+}$over $T_{1}$ values in the search range $(\mathrm{B})$, the maximum $\tilde{\sigma}_{T_{1}}$ for each $B_{1}^{+}$over $T_{1}$ values in the search range $(\mathrm{C})$, and the maximum $\tilde{\sigma}_{T_{1}}$ for each $T_{1}$ over $B_{1}^{+}$values in the search range (D).
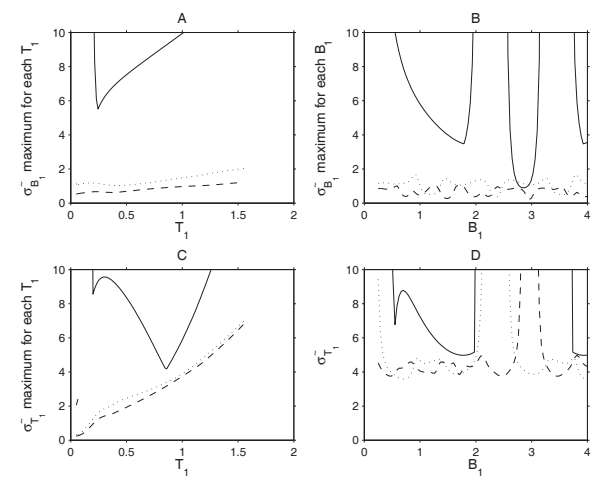

Fig. 2. AFI model, compare Fig. 1.
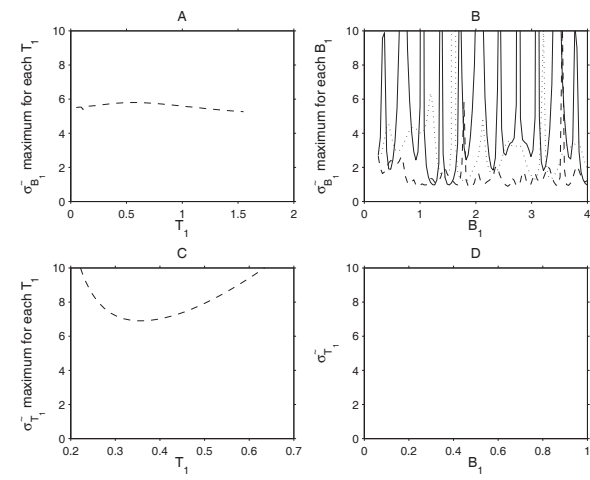

Fig. 3. BP model, compare Fig. 1. 


\subsection{Discussion}

In this analysis, we consider two main questions: 1) What is the trade off between $\tilde{\sigma}_{b}$ and $\tilde{\sigma}_{T_{1}}$ ? and 2) How robust are the optimal parameters found in (10)?

Fig. 4 and Fig. 5 show the trade off between $\tilde{\sigma}_{b}^{\max }$ and $\tilde{\sigma}_{T_{1}}^{\max }$. Improved accuracy in estimating $B_{1}^{+}$decreases $T_{1}$ accuracy. Therefore, in scan parameter optimization, a function of both TRCDs is required. The SSI and AFI method have the lowest achievable worst case TRCD (the BP method is outside Fig. 4). both the AFI and SSI method perform well for $N=4$ and $N=8$, with the AFI method having a slight advantage. For $N=2$ (not shown), the SSI has a clear advantage.

The optimal parameters robustness varies both on the method and the number of scans (see Figures Fig. 1, Fig. 2, and Fig. 3). TRCD, for all methods, is lowest when $T_{1}$ is small (plots A and C), but is more robust to the value of $B_{1}^{+}$ (plots B and D). This is especially true for $\tilde{\sigma}_{T_{1}}$. For all methods, $N=4,8$ performs much better than $N=2$, especially for the AFI method. Using four or eight scans, both the SSI and AFI method are relatively insensitive to specific values of $B_{1}^{+}$and $T_{1}$ and are appropriate to use for joint estimation, though SSI has the lowest TRCD values consistently. The BP method has relatively high TRCD values, even when $N=8$, and $\tilde{\sigma}_{b}$ is especially sensitive to the value of $B_{1}^{+}$, so this method as implemented will have high variance for unbiased $B_{1}^{+}$estimation.

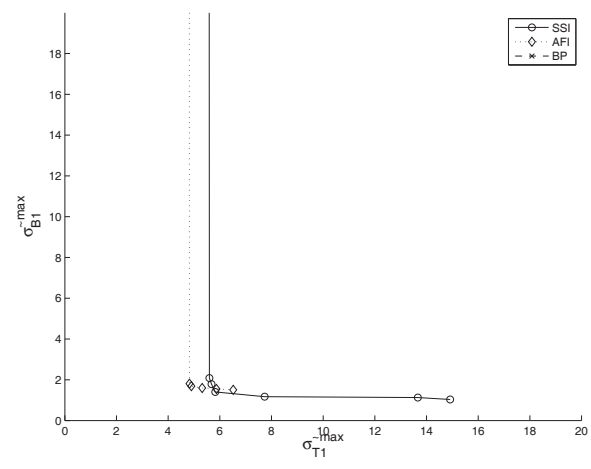

Fig. 4. Minimum achievable $\tilde{\sigma}_{b}^{\max }$ for a maximum $\tilde{\sigma}_{T_{1}}^{\max }$ for two scans.

\section{CONCLUSIONS}

After analyzing the CRB for joint estimation of $B_{1}^{+}$and $T_{1}$, the SSI has both the lowest worst case estimator variances and is the least sensitive to $B_{1}^{+}$and $T_{1}$ values. The AFI is also relatively insensitive to $B_{1}^{+}$and $T_{1}$ values, but, overall, has higher estimator variances. The BP model, as modeled here, exhibits the highest optimized variance, although this may be improved by further optimizing other scan parameters in the model. Although the results are not shown here, we also

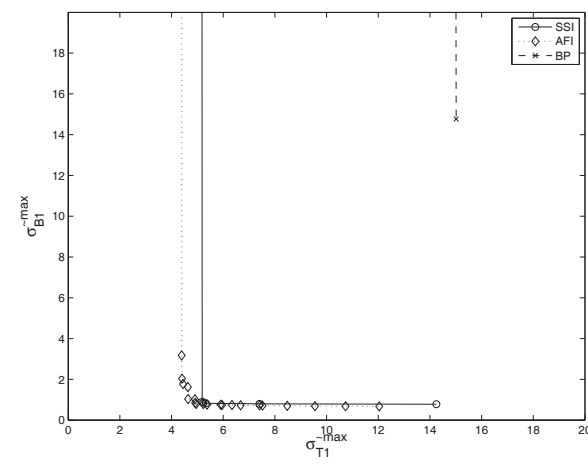

Fig. 5. Minimum achievable $\tilde{\sigma}_{b}^{\max }$ for a maximum $\tilde{\sigma}_{T_{1}}^{\max }$ for eight scans.

tried using the SSI model and varying $T_{R}$, but had very poor results.

We note that this optimization does neglect SAR constraints which may be a problem when using a large tip angle and a short repetition time. The effect of $B_{0}$ is also neglected in the models (7), (8), and (9). The effect of considering much lower $T_{R},($ e.g., $10 \mathrm{~ms})$, on the CRB is also not considered. These will be further analyzed in the future work.

\section{REFERENCES}

[1] U. Katscher, P. Brnert, C. Leussler, and J. S. van den Brink, “Transmit SENSE," Mag. Res. Med., vol. 49, no. 1, pp. 144-50, Jan. 2003.

[2] C. H. Cunningham, J. M. Pauly, and K. S. Nayak, "Saturated double-angle method for rapid $B_{1}+$ mapping," Mag. Res. Med., vol. 55, no. 6, pp. 1326-33, June 2006.

[3] D. O. Brunner and K. P. Pruessmann, "B1+ interferometry for the calibration of RF transmitter arrays," Mag. Res. Med., vol. 61, no. 6, pp. 1480-8, June 2009.

[4] H-L. M. Cheng and G. A. Wright, "Rapid high-resolution $T_{1}$ mapping by variable flip angles: Accurate and precise measurements in the presence of radiofrequency field inhomogeneity," Mag. Res. Med., vol. 55, no. 3, pp. 566-74, Mar. 2006.

[5] E. M. Haacke, R. W. Brown, M. R. Thompson, and R. Venkatesan, Magnetic resonance imaging: Physical principles and sequence design, Wiley, New York, 1999.

[6] S. C. L. Deoni, B. K. Rutt, and T. M. Peters, "Rapid combined T1 and T2 mapping using gradient recalled acquisition in the steady state," Mag. Res. Med., vol. 49, no. 3, pp. 515-26, Mar. 2003.

[7] N. G. Dowell and P. S. Tofts, "Fast, accurate, and precise mapping of the RF field in vivo using the $180^{\circ}$ signal null," Mag. Res. Med., vol. 58, no. 3, pp. 622-30, Sept. 2007.

[8] V. L. Yarnykh, "Actual flip-angle imaging in the pulsed steady state: A method for rapid three-dimensional mapping of the transmitted radiofrequency field," Mag. Res. Med., vol. 57, no. 1, pp. 192-200, Jan. 2007. 